\title{
Promotion of Liver and Lung Tumorigenesis in DEN-Treated Cytoglobin-Deficient Mice
}

\author{
Le Thi Thanh Thuy, ${ }^{* \dagger}$ Takashi Morita, ${ }^{\ddagger}$ \\ Kayo Yoshida, ${ }^{\ddagger}$ Kenichi Wakasa, ${ }^{\S}$ \\ Masashi lizuka, ${ }^{\star \dagger}$ Tomohiro Ogawa, ${ }^{* \dagger}$ Mami Mori, ${ }^{*}$ \\ Yumiko Sekiya, ${ }^{* \dagger}$ Shinobu Momen, ${ }^{* \dagger}$ \\ Hiroyuki Motoyama, ${ }^{*}$ Kazuo Ikeda, ${ }^{\text {" }}$ \\ Katsutoshi Yoshizato, ${ }^{*+\|}$ and Norifumi Kawada ${ }^{\star \dagger}$ \\ From the Departments of Hepatology, ${ }^{*}$ Molecular Genetics, ${ }^{*}$ and \\ Diagnostic Pathology, ${ }^{\S}$ and the Liver Research Center, ${ }^{\dagger}$ Graduate \\ School of Medicine, Osaka City University, Osaka; the \\ Department of Anatomy and Cell Biology, "Graduate School of \\ Medical Sciences, Nagoya City University, Aichi; and the \\ Academic Advisor's Office," PhoenixBio Co., Ltd., Hiroshima, Japan
}

Cytoglobin (Cygb) is a recently discovered vertebrate globin with molecular characteristics that are similar to myoglobin. To study the biological function of Cygb in vivo, we generated Cygb knockout mice and investigated their susceptibility to $N, N$-diethylnitrosamine (DEN)-induced tumorigenesis. Four-week-old male mice were administered DEN in drinking water at a dose of $25 \mathrm{ppm}$ for 25 weeks or $0.05 \mathrm{ppm}$ for 36 weeks. Cygb deficiency promoted the DEN-induced development of liver and lung tumors. All $\mathrm{Cygb}^{+/-}$ and $C y g b^{-1-}$ mice treated with 25-ppm DEN exhibited liver tumors, compared with $44.4 \%$ of their wild-type counterparts. Lung tumors were present only in Cygbdeficient mice. More than $40 \%$ of $\mathrm{Cygb}^{-/-}$mice developed liver and lung tumors at the nontoxic dose of DEN (0.05 ppm), which did not induce tumors in wild-type mice. Cygb loss was associated with increased cancer cell proliferation, elevated extracellular signal-regulated kinase and Akt activation, overexpression of IL-1 $\beta$, IL-6, Tnf $\alpha$, and Tgf $\beta 3$ mRNAs, and hepatic collagen accumulation. Cygb-deficient mice also exhibited increased nitrotyrosine formation and dysregulated expression of cancer-related genes (cyclin D2, p53, Pak1, Src, Cdkn2a, and Cebpa). These results suggest that $C y g b$ deficiency induces susceptibility to cancer development in the liver and lungs of mice exposed to DEN. Thus, globins such as Cygb will shed new light on the biological features of organ carcinogenesis. (Am J Pathol 2011, 179:1050-1060; DOI: 10.1016/j.ajpath.2011.05.006)
Cytoglobin (Cygb) was originally identified in 2001 as a protein up-regulated in rat hepatic stellate cells under profibrotic conditions. Accordingly, Cygb was originally termed a stellate cell activation-associated protein ${ }^{1}$ until it was identified as the fourth globin in mammals. ${ }^{2,3} \mathrm{Hu}-$ man Cygb displays approximately $25 \%$ amino acid identity with vertebrate myoglobin and hemoglobin and $16 \%$ identity with human neuroglobin. The Cygb gene is localized to chromosome 17q25.3 in humans and chromosome 11E2 in mice.

Unlike myoglobin, which is tissue restricted to cardiomyocytes and skeletal myofibers, hemoglobin in erythrocytes, and neuroglobin in the nervous system, Cygb is ubiquitously expressed in the cytoplasm of mesenchymal fibroblastic cells in many organs, including the heart, lung, liver, kidneys, small intestine, and spleen. The presence of Cygb in the nucleus of these cells has also been reported. ${ }^{4,5}$ In particular, Cygb was present in stellate cells and myofibroblasts in the liver and pancreas, reticulocytes in the spleen, mesenchymal cells in the submucosal layer of the gut, and the mesangium and stromal cells of the kidney. ${ }^{4}$ An interesting aspect of Cygb expression is its presence in visceral cells, with a strong storage ability for vitamin A. Thus, Cygb may facilitate the diffusion of oxygen through tissues, scavenge nitric oxide (NO) or other reactive oxygen species, or serve a protective function during oxidative stress. ${ }^{6}$ However, the precise physiological role of Cygb in vivo remains unresolved. Cygb is considered a hypoxia-responsive molecule because its mRNA expression is augmented under hypoxia in fibroblastic cell lineages and rat brain. ${ }^{7}$ Hypoxiainducible factor 1 is assumed to be an important transcription factor for Cygb because hypoxia-responsive elements at positions $-141,-144$, and -448 are essential for the acti-

Supported by a Grant-in-Aid for Scientific Research from the Japan Society for the Promotion of Science [grant 21390232 (2009) to N.K.]; grants from the Ministry of Health, Labour, and Welfare of Japan (2008); and the Thrust Area Research grant from Osaka City University (2008).

Accepted for publication May 2, 2011

Presented in part at the $16^{\text {th }}$ International Symposium on Cells of the Hepatic Sinusoid, Pasadena, CA, September 2, 2010.

Address reprint requests to Norifumi Kawada, M.D., Ph.D., Department of Hepatology, Graduate School of Medicine, Osaka City University, 1-4-3 Asahimachi, Abeno, Osaka 545-8585, Japan. E-mail: kawadanori@med. osaka-cu.ac.jp. 
vation of CYGB gene expression, and the binding of hypoxia-inducible factor 1 to this area has been confirmed. ${ }^{8,9}$ In contrast, CYGB overexpression rescues the human neuronal cell line TE671 from prooxidant Ro19-8022-induced DNA damage. ${ }^{10} \mathrm{CYGB}$ overexpression also protected human neuroblastoma SH-SY5Y cells from $\mathrm{H}_{2} \mathrm{O}_{2}$-induced cell death. ${ }^{11,12}$ Furthermore, the in vitro and in vivo overexpression of Cygb in rat hepatic stellate cells protected these cells against oxidative stress and inhibited their differentiation into an active phenotype. ${ }^{13}$ Together, these reports suggest that Cygb may act as a cytoprotective and radicalscavenging molecule in addition to its function as a gas carrier.

Although the function of Cygb in vivo remains largely unknown, down-regulation of CYGB has been reported in several human cancerous tissues and human cancer cell lines. Decreased expression of CYGB and the hypermethylation of the CYGB promoter has been reported in patients with tylosis, non-small-cell lung carcinoma tissues, head and neck cancers, ovarian cancers, and breast cancers. ${ }^{14-18}$ McRonald et $\mathrm{al}^{14}$ reported that CYGB gene expression in tylosis with esophagus cancer was reduced to approximately $70 \%$ compared with the normal esophagus and was accompanied by hypermethylation of the promoter. Xinarianos et $\mathrm{a}^{15}$ reported a significant reduction of CYGB mRNA expression in non-small-cell lung carcinoma tissues and hypermethylation of CYGB, compared with healthy samples. Similar results were reported in head and neck, ovarian, and breast cancer tissues. ${ }^{16-18}$ In addition, Shivapurkar et al ${ }^{19}$ reported high levels of CYGB promoter methylation in lung, breast, bladder, and colon cancers and in leukemia in humans. The augmented growth of $\mathrm{NCl}-\mathrm{H} 661$ lung cancer cells that were CYGB silenced by RNA interference and the suppression of $\mathrm{NCl}-\mathrm{H} 228$ cell proliferation in cells stably transfected with plasmids containing CYGB cDNA have also been reported. ${ }^{19}$ These reports indicate a tumor suppressor function of Cygb.
To study the biological function of Cygb at the tissue level, we first generated Cygb-deficient $\left(\mathrm{Cygh}^{-1-}\right)$ mice and observed that, after treatment with $\mathrm{N}, \mathrm{N}$-diethylnitrosamine (DEN), Cygb ${ }^{-1-}$ mice showed a high incidence of tumor development in the liver and lungs. These results indicate the tumor suppressor role of Cygb in vivo.

\section{Materials and Methods}

\section{Materials}

All experimental reagents were obtained from Sigma Chemical Co (St. Louis, MO) or Wako Pure Chemical Co (Osaka, Japan), unless otherwise stated.

\section{Construction of the Targeting Vector}

Mice lacking exon 1 of the Cygb gene were generated using the lox-P system, as previously described. ${ }^{20}$ The targeting vector (pTVneo/Cygb) was constructed from PCR DNA fragments from 129Sv mouse genomic DNA. Two DNA fragments were used as the $3^{\prime}$ and $5^{\prime}$ arms. One fragment contained Cygb exons 2, 3, and 4 (6.5 $\mathrm{kb})$; and the other contained the transcription initiation site in exon 1 and the $5^{\prime}$ upstream sequence of the Cygb gene $(6.0 \mathrm{~kb})$. The neomycin-resistance gene, driven by the phosphoglycerate kinase 1 promoter, flanking the lox-P sequences was inserted into the arms (Figure 1A).

Embryonic stem cells $\left(1 \times 10^{7}\right.$ cells $\left./ \mathrm{mL}\right)$ were transfected with a linearized targeting vector $(20 \mu \mathrm{g})$ by electroporation and cultured in selection medium containing $150 \mu \mathrm{g} / \mathrm{mL}$ geneticine (G418). Of 480 neomycin-resistant clones, $6(1.2 \%)$ were homologous recombinants by Southern blotting using the $5^{\prime}$ probe (data not shown).
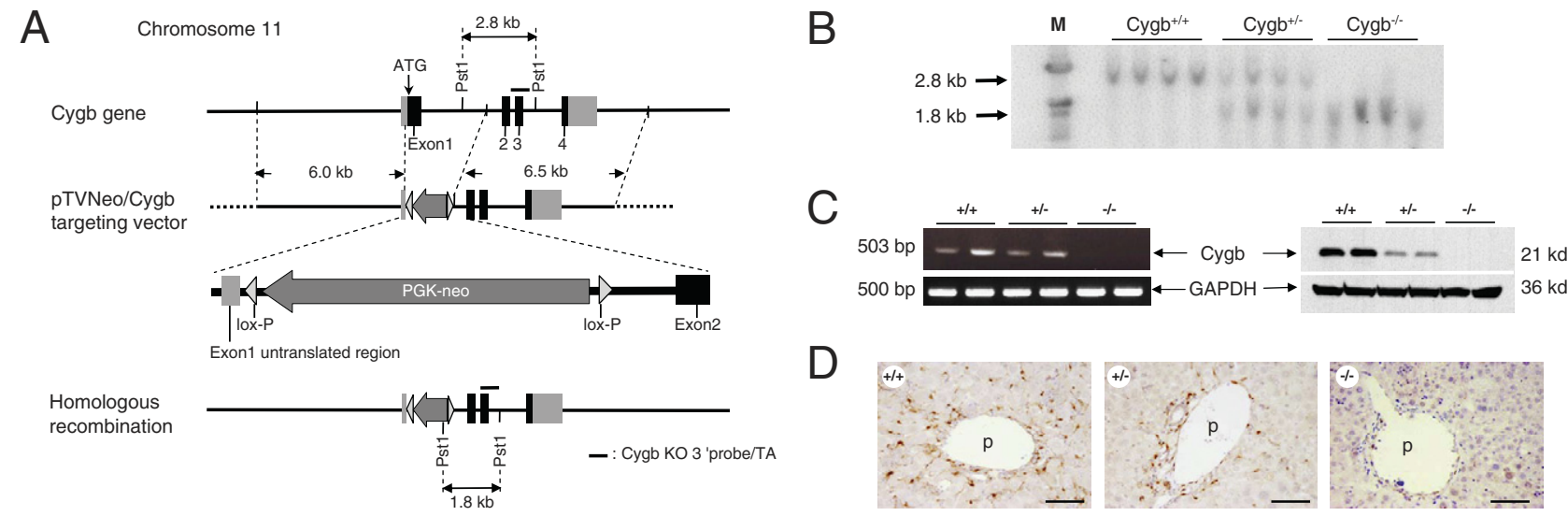

D
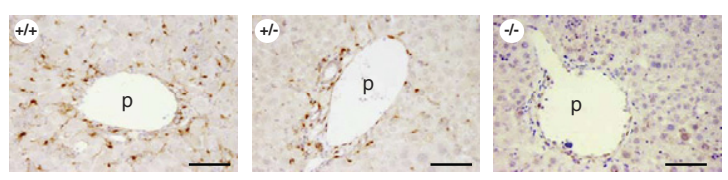

Figure 1. Generation of Cygb-deficient mice. A: Strategy for inactivation of the Cygb gene by homologous recombination in embryonic stem cells. A partial genomic map of the Cygb gene with coding exons (black boxes), noncoding regions (light gray boxes), and flanking introns (solid lines) is shown (top). Targeting vector pTVneo/Cygb with homology to the Cygb gene locus is shown (middle). The translation initiation site in exon 1 was replaced with a neomycin-resistance cassette. The predicted Cygb gene locus after homologous recombination is shown (bottom). B: Southern blot analysis shows the sizes of the wild-type (2.8-kb) and disrupted (1.8-kb) Cygb fragments after PstI cleavage. M, molecular weight marker. C: RT-PCR and immunoblot analysis of Cygb expression in the liver from $C y g b^{+/+}, C y g b^{+/-}$, and $C y g b^{-1-}$ mice. GAPDH was used as a loading control. D: Representative IHC images of Cygb in the liver of $\mathrm{Cygb}^{+/+}, \mathrm{Cygh}^{+/-}$, and $C y g b^{->-}$mice. Cygb is present along the sinusoids of $C y g b^{+/+}$mice, whereas it is undetectable in $C y g b^{-/-}$mice. p, portal vein. Scale $\mathrm{bar}=50 \mu \mathrm{m}$. 


\section{Production of Cygb-Deficient Mice}

Two clones were aggregated with C57BL/6-DBA2 F1 mouse morulae, and one produced chimeric mice that transmitted the knockout (KO) construct. Chimeric males were mated with $\mathrm{C} 57 \mathrm{BL} / 6 \mathrm{~J}$ females to obtain Cygb heterozygous mice that were backcrossed to the C57BL/6J background for more than nine generations. To assess the role of the Cygb gene in development, we intercrossed Cygb heterozygous mice. The litter sizes were normal, and analysis of the tail biopsy specimens at the age of 4 weeks, from 102 offspring from heterozygote crosses, revealed the presence of homozygous mutant mice at a frequency of $24 \%$. The homozygotes appeared normal morphologically and histopathologically 1 month after birth. Four-week-old $\mathrm{Cygh}^{+/+}$(wild-type), Cygh ${ }^{+/-}$(heterozygote), and $\mathrm{Cygh}^{-1-}$ (homozygote) male mice were used in this study.

All mice were cared for according to the guidelines approved by the Institutional Animal Care and Use Committee of Osaka City University, Osaka.

\section{Genotyping of Mice}

PCR genotyping of mouse tail DNA produced the expected 338-bp product from wild-type mice using the following primer pairs: forward, 5'-CTCCCAGCCGGGACCGCGGTGGCCTT-3'; and reverse, 5'-GGAGCCGAGGCCGGTGCGTGCGAGGC-3'. A 529-bp product was diagnostic for the Cygb KO allele using the described forward primer and the following reverse primer: 5'-GTGGGGTGGGATTAGATAAATGCCTGCTCT-3'. PCR was performed in a $15-\mu \mathrm{L}$ reaction mixture containing $1 \mu \mathrm{L}$ of extract from mouse tail DNA, which was digestion extracted using LYPPO (Gene Modification R\&D Co Ltd, Osaka), according to the manufacturer's protocol; $1 \mu \mathrm{mol} / \mathrm{L}$ of each primer; $2.5 \%$ to $5 \%$ dimethyl sulfoxide; and $0.5 \cup$ GO Taq polymerase (Promega, San Luis Obispo, CA). PCRs were performed for 40 cycles, each cycle being 1 minute at $94^{\circ} \mathrm{C}, 30$ seconds at $70^{\circ} \mathrm{C}$, and 1 minute at $72^{\circ} \mathrm{C}$.
Southern blot analysis confirmed the Cygb-null allele. Genomic tail DNA (5 $\mu \mathrm{g})$ was cleaved with Pstl, subjected to agarose gel electrophoresis, blotted onto nylon membranes, and hybridized to the ${ }^{32} \mathrm{P}$-labeled Cygb $\mathrm{KO} 3^{\prime}$ probe/TA (Figure 1A). DNA fragments of 2.8 and $1.8 \mathrm{~kb}$ represented the Cygb wild-type and null alleles, respectively.

RT-PCR was performed to confirm the absence of Cygb mRNA in tissues. Total RNA was extracted from the homogenates of liver tissues using the RNeasy Mini Kit (Qiagen, Valencia, CA). cDNA was synthesized using 1 $\mu \mathrm{g}$ of total RNA, ReverTra Ace (Toyobo, Osaka), and oligo (dT) ${ }_{12-18}$ primers, according to the manufacturer's instructions. Thirty-five PCR cycles $\left(30\right.$ seconds at $94^{\circ} \mathrm{C}$, 30 seconds at $68^{\circ} \mathrm{C}$, and 1 minute at $72^{\circ} \mathrm{C}$ ) were run using the following mouse Cygb primers: forward, 5'GCGACATGGAGATAGAGCGT-3'; and reverse, 5'-CTGTACCCAGCCCACTTCCT-3'. This generated a 503-bp product and glyceraldehyde-3-phosphate dehydrogenase (GAPDH) primers: forward, 5'-CGCCTGGTCACCAGG-3'; and reverse, 5'-CAGTTGGTGGTGCAGGA$\left.3^{\prime}\right)$. A 500-bp product was generated.

\section{Administration of DEN}

DEN $(0.95 \mathrm{~g} / \mathrm{mL})$ was obtained from Sigma Chemical Co. A stock solution of DEN was prepared by dissolving $1 \mathrm{~g}$ (1.06 mL) of DEN in $400 \mathrm{~mL}$ of water. The stock solution was diluted 100 -fold before use to obtain a final concentration of 25-mg DEN per 1000-mL water (25 ppm) for the high-dose experiment. The 25-ppm solution was further diluted 500 -fold to obtain a final concentration of $0.05-\mathrm{mg}$ DEN per $1000-\mathrm{mL}$ water $(0.05 \mathrm{ppm})$ for the low-dose experiment. The diluted solution ( 25 or $0.05 \mathrm{ppm}$ ) was placed in a shaded serving bottle and administered to the animals instead of water. The diluted solution was prepared weekly. The administration of DEN to male mice began at the age of 4 weeks for 25 weeks in the highdose experiment (25 ppm) and for 36 weeks in the lowdose experiment ( $0.05 \mathrm{ppm})$. Each experiment contained three groups $\left(\mathrm{Cygb}^{+/+}, \mathrm{Cygh}^{+/-}\right.$, and $\left.\mathrm{Cygh}^{-1-}\right)$ with a

Table 1. Primary Antibodies Used for IHC Analyses

\begin{tabular}{llll}
\hline \multicolumn{1}{c}{ Antigen* } & \multicolumn{1}{c}{ Source } & \multicolumn{1}{c}{ Name/clone; catalog no. } & \multicolumn{1}{c}{ Incubation } \\
\hline AFP & US Biological, Swampscott, MA & F4100-16A (Go) & Overnight $4^{\circ} \mathrm{C}, 1: 20$ \\
CK19 & Santa Cruz Biotechnology, & Polyclonal (Rb); sc-33111 & Overnight $4^{\circ} \mathrm{C}, 1: 100$ \\
& Santa Cruz, CA & & \\
CRBP-1 & Santa Cruz Biotechnology & Polyclonal (Rb); sc-30106 & Overnight $4^{\circ} \mathrm{C}, 1: 100$ \\
Cygb & Our laboratory ${ }^{+}$ & Polyclonal (Rb) & Overnight $4^{\circ} \mathrm{C}, 1: 100$ \\
Erk & Cell Signaling, Danvers, MA & Monoclonal (Rb); 4695 & Overnight $4^{\circ} \mathrm{C}, 1: 300$ \\
PCNA & Dako, Glostrup, Denmark & Monoclonal (Mo) ${ }^{\ddagger} ;$ clone: PC-10 & Overnight $4^{\circ} \mathrm{C}, 1: 200$ \\
Phosphorylated & Cell Signaling & Monoclonal (Rb); 4370 & Overnight $4^{\circ} \mathrm{C}, 1: 200$ \\
Erk & Dako & Monoclonal (Mo) $)^{\ddagger} ;$ clone: $1 \mathrm{~A} 4$ & 30 minutes at room temperature, $1: 100$ \\
$\alpha$-SMA & Santa Cruz Biotechnology & Polyclonal (Go); sc-7559 & Overnight $4^{\circ} \mathrm{C}, 1: 100$ \\
Desmin & &
\end{tabular}

${ }^{*}$ All antigens were retrieved by autoclaving for 15 minutes in $0.01 \mathrm{~mol} / \mathrm{L}$ citrate buffer containing $0.05 \%$ Tween 20 (pH 6.0), except for desmin, which was used in Tris-EDTA buffer ( $\mathrm{pH}$ 9.0).

${ }^{\dagger}$ Data taken from Kawada et al. ${ }^{1}$

\#For mouse primary antibodies, after antigen retrieval, sections were incubated with goat anti-mouse IgG Fab fragments (Jackson ImmunoResearch Laboratories) for 1 hour at room temperature (1:100) to block nonspecific background staining.

AFP, alpha-fetoprotein. 
minimum of seven mice per group. Mice were sacrificed after the completion of DEN treatment.

\section{Necropsy}

At necropsy, mice were weighed, anesthetized, and examined for grossly visible lesions in whole organs. Livers and lungs were excised, weighed (in the case of liver), and examined for macroscopic lesions. The number of macroscopic abnormal masses $\geq 1 \mathrm{~mm}$ was determined in addition to the size of the mass by taking the average of the largest and smallest length of the mass. For histological examination, 2- to 3-mm-thick sections from nontumor or tumor tissues were fixed in 10\% formalin for 24 hours and embedded in paraffin. The samples were then sectioned at $5 \mu \mathrm{m}$ and stained with $\mathrm{H} \& \mathrm{E}$.

\section{IHC and TUNEL Assays}

For immunohistochemistry (IHC), paraffin sections were dewaxed in xylene and rehydrated in decreasing concentrations of ethanol. The primary antibodies and conditions used for IHC are listed in Table 1. Negative controls with no primary antibody were used to assess nonspecific staining. The secondary antibodies used were horseradish peroxidase-conjugated goat anti-rabbit IgG (1:200; Dako, Glostrup, Denmark), rabbit anti-goat IgG (1:200; Dako), or rabbit anti-mouse IgG (1:200; Dako). 3,3'-Diaminobenzidine (Dako) was used as the chromogen. TdT-mediated dUTP-biotin nick-end labeling (TUNEL) staining was performed using the In situ Apoptosis Detection Kit (MK500; TaKaRa Bio Inc., Shiga, Japan), according to the manufacturer's protocol. All sections were counterstained with Meyer's hematoxylin.

\section{Quantification of Liver Fibrosis}

Morphometric image analysis was performed in liver tissue specimens with a computerized system, consisting of a photomicroscope, a digital camera, and LuminaVision 2.4 bioimaging software (Mitani Corporation, Tokyo, Japan) to quantitatively assess fibrosis. The proportion of the area stained with Sirius red in the liver sections was calculated as the sum of the pixelwise-bound stain measurements divided by the number of summed pixels.

\section{Quantitative Real-Time PCR}

Total RNA was extracted from liver and liver tumor tissues using the RNeasy Mini Kit (Qiagen, Valencia, CA). cDNAs were synthesized as previously described. Gene expression was measured by quantitative real-time PCR using cDNA, real-time PCR Master Mix Reagents (Toyobo, Osaka), or TaqMan Fast Universal PCR Master Mix (Applied Biosystems, Foster City, CA), and a set of genespecific oligonucleotide primers and probes (Table 2) using an Applied Biosystems Prism 7500 (Applied Biosystems). GAPDH levels were measured and used to normalize the relative abundance of mRNA.
Table 2. Primers Used for RT-qPCR

\begin{tabular}{|c|c|}
\hline Gene & Sequence \\
\hline \multicolumn{2}{|l|}{ AFP } \\
\hline Forward & $5^{\prime}-$ САCACCCGCTTCCCTCAT-3' \\
\hline Reverse & 5'-TTTTCGTGCAATGCTTTGGA-3' \\
\hline \multicolumn{2}{|r|}{ 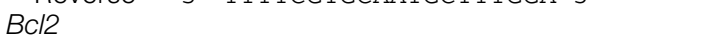 } \\
\hline Forward & 5'-AAGGGCTTCACACCCAAATCT-3' \\
\hline Reverse & $5^{\prime}$-CTTCTACGTCTGCTTGGCTTTGA-3' \\
\hline \multicolumn{2}{|r|}{ 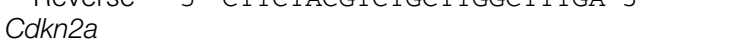 } \\
\hline Forward & 5'-GCTCTGGCTTTCGTGAACATG-3' \\
\hline Reverse & 5'-GTGCGGCCCTCTTCTCAA-3' \\
\hline \multicolumn{2}{|r|}{ (5) } \\
\hline Forward & $5^{\prime}-$ CGCAAGAGCCGAGATAAAGC- $3^{\prime}$ \\
\hline Reverse & 5'-CGGTCATTGTCACTGGTCAACT-3' \\
\hline \multicolumn{2}{|l|}{ Col1 $\alpha 1$} \\
\hline Forward & 5'-GACATCCCTGAAGTCAGCTGC-3' \\
\hline Reverse & 5'-TCCCTTGGGTCCCTCGAC-3' \\
\hline \multicolumn{2}{|l|}{ Cyclin D1 } \\
\hline Forward & $5^{\prime}-$ GCCCGGAGGGATTTGC-3' \\
\hline Reverse & 5'-AGACGGAACACTAGAACCTAACAGATT-3' \\
\hline \multicolumn{2}{|r|}{ כ -AGACGGAACACTAGAACL TAACAGATI- ? } \\
\hline Forward & $5^{\prime}$-AAGGCAGATACTCATCAAACACAGA-3' \\
\hline \multirow{2}{*}{\multicolumn{2}{|c|}{ J - LIGGIGLALGLAGLAA-3 }} \\
\hline & \\
\hline Forward & 5'-CCCCAAACTTCGACCATGAT-3' \\
\hline Reverse & 5'-GGAGGATGACGCCTCGTAGTC-3' \\
\hline \multicolumn{2}{|l|}{ GAPDH } \\
\hline Forward & $5^{\prime}-$ TGCACCACCAACTGCTTAG- $3^{\prime}$ \\
\hline Reverse & $5^{\prime}$-GGATGCAGGGATGATGTTC-3' \\
\hline \multicolumn{2}{|r|}{ 5 -GGATGLAGGGATGATGILL-3 } \\
\hline Forward & 5'-CGCTATGAAGTTCCTCTCTGCAA-3' \\
\hline Reverse & $5^{\prime}$-CACCAGCATCAGTCCCAAGA- $3^{\prime}$ \\
\hline \multicolumn{2}{|r|}{ gere } \\
\hline Forward & 5'-CCATGGCACATTCTGTTCAAA-3' \\
\hline Reverse & $5^{\prime}-$ GCCCATCAGAGGCAAGGA- $3^{\prime}$ \\
\hline \multicolumn{2}{|r|}{5 -GLCLATLAGAGGLAAGGA-3 } \\
\hline Forward & $5^{\prime}-\mathrm{CCGCCCCTGTCCCCTAT-3^{ \prime }}$ \\
\hline Reverse & 5'-TCCTCATGCGCTTCCTCTCT-3' \\
\hline \multicolumn{2}{|l|}{ Pak1 } \\
\hline Forward & 5'-CGTATTGCGGGTGTTTGCTA-3' \\
\hline \multirow{2}{*}{\multicolumn{2}{|c|}{5 - CACAGLAGGAGAACLAAAACL - 3}} \\
\hline & \\
\hline Forward & $5^{\prime}-$ GCATGAACCGCCGACCTAT-3' \\
\hline Reverse & 5'-CAGAAGGTTCCCACTGGAGTCT-3' \\
\hline \multicolumn{2}{|r|}{ 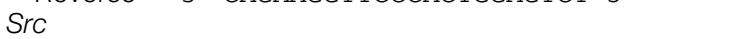 } \\
\hline Forward & $5^{\prime}-\mathrm{CCTCCCGCACCCAGTTC-3^{ \prime }}$ \\
\hline Reverse & 5'-CATCAGCATGTTTGGAGTAGTAAGC-3' \\
\hline \multicolumn{2}{|r|}{ J -CATLAGLATGIIIGGAGTAGIAAGL-3 } \\
\hline Forward & $5^{\prime}-$ AGGGCCCTGGACACCAATTAC-3' \\
\hline Reverse & $5^{\prime}$-CCTTAGGTTCTGGGACCCATTTC-3' \\
\hline \multicolumn{2}{|r|}{ 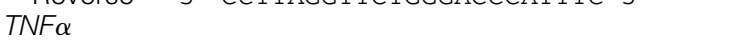 } \\
\hline Forward & 5'-ССТСАСАСТСАGАTCTTCTCA-3' \\
\hline Reverse & 5'-GCTGCTCCTCCACTTGGTG-3' \\
\hline Probe & $5^{\prime}$-GCAAGCCTGTAGCCCACGTCGTAGCAAA-3' \\
\hline
\end{tabular}

\section{Immunoblotting}

Protein samples (10 to $20 \mu \mathrm{g}$ ) were subjected to SDSPAGE and transferred to Immobilon P membranes (Millipore Corp, Bedford, MA). After blocking, membranes were probed with primary antibodies against Cygb (1: 500) from our laboratory (Table 1), Akt (1:1000; Cell Signaling, Danvers, MA), phosphorylated Akt (1:500; Cell Signaling), extracellular signal-regulated kinase (Erk; 1:500; Cell Signaling), phosphorylated Erk (1:1000; Cell Signaling), cyclin D1 (1:5000; Cell Signaling), nitrotyrosine (1:500; Cell Signaling), or GAPDH (1:2000; Santa Cruz Biotechnology, Santa Cruz, CA). Membranes were 
then labeled with horseradish peroxidase-conjugated secondary antibodies. Immunoreactive bands were visualized using the ECL detecting reagent (GE Healthcare UK Ltd, Buckinghamshire, UK) and documented with an LAS 1000 (Fuji Photo Film, Kanagawa, Japan).

\section{Data Analyses}

The data presented as bar graphs are the means \pm SDs in all experiments. Statistical analyses were performed using the Student's $t$-test, and $P<0.05$ indicated statistical significance.

\section{Results}

\section{Characterization of the Cygb ${ }^{-1-}$ Mice}

Cygb-deficient mice were generated by deleting exon 1 of the mouse Cygb gene (Figure 1A) and backcrossed on the C57BL/6J background. Southern blotting (Figure 1B) and PCR genotyping using mouse tail DNA (data not shown) confirmed the deletion of the Cygb gene. Both Cygb mRNA and protein expression were absent in $\mathrm{Cygh}^{-1-}$ mouse livers, compared with wild-type livers (Figure 1C). Furthermore, IHC indicated that the Cygb protein was present in sinusoidal lining cells in addition to perivascular cells around the portal and central veins, as previously reported, ${ }^{1}$ in $\mathrm{Cygh}^{+/+}$mice, whereas it was undetected in $\mathrm{Cygb}^{-/-}$ mice (Figure 1D). Together, these data demonstrate the successful production of the $\mathrm{Cygh}^{-1-}$ mice.

The mice homozygous for the disrupted allele appeared normal both morphologically and histopathologically 1 month after birth. Next, we examined whether Cygb influenced the toxicity and carcinogenesis of DEN in mice.

\section{Cygb Deficiency Strongly Promotes DEN-Induced Tumorigenesis}

DEN is a commonly used chemical carcinogen for the liver because it is activated by cytochrome P-450 enzymes in hepatocytes. ${ }^{21} \mathrm{C} 57 \mathrm{BL} / 6 \mathrm{~J}$ mice were reported to be relatively resistant to liver tumor development under DEN treat-

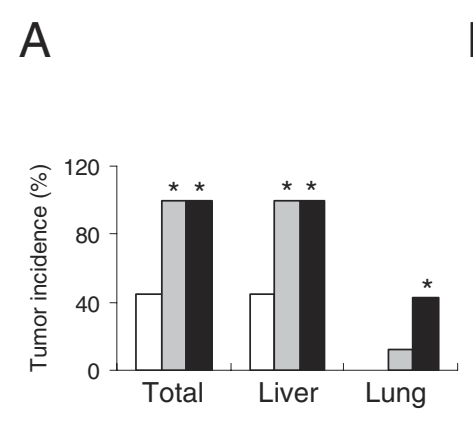

C

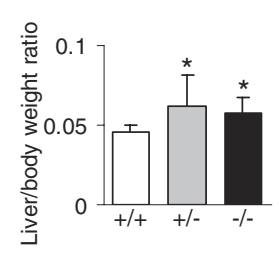

D

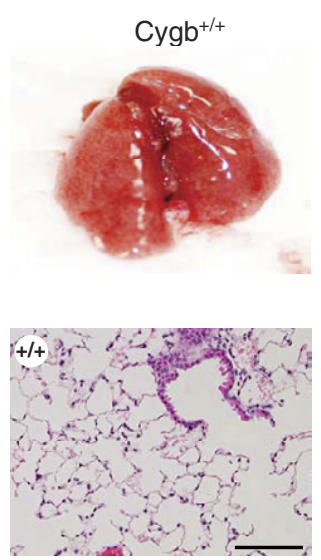

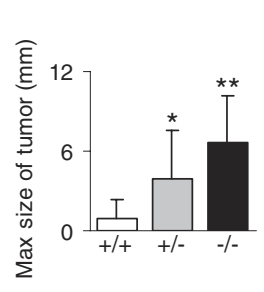
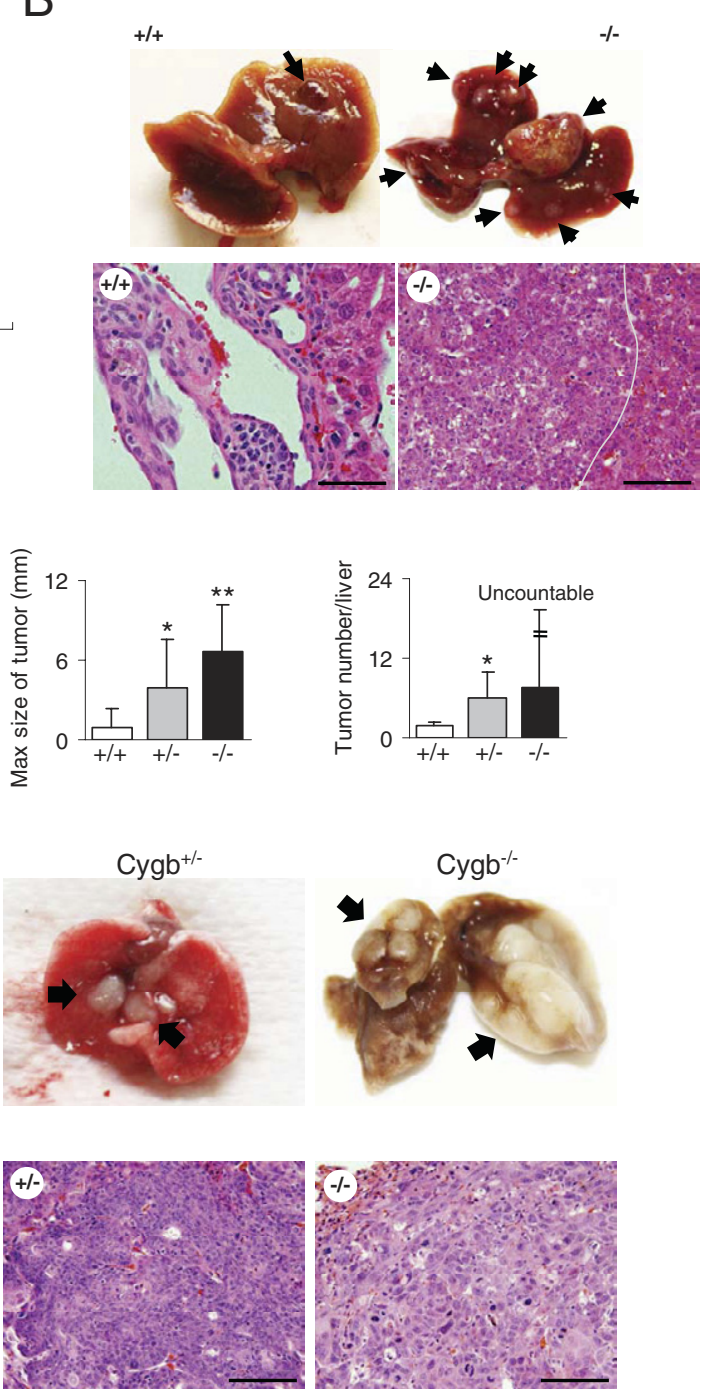

Figure 2. Tumor development in Cygb-deficient mice treated with 25-ppm DEN for 25 weeks. A: Tumor incidence in total, livers, and lungs from DEN-treated $C y g b^{+/+}$(white bars), $\mathrm{Cygb}^{+/-}$(gray bars), and $\mathrm{Cygb}^{-1-}$ (black bars) mice ( $n=7$ to 12 ). ${ }^{*} P<0.05$ compared with wild type. B: Representative gross photographs of livers (top) from wild-type $(+/+)$ and Cygb-KO $(-/-)$ mice treated with DEN. There was a marked increase in tumor multiplicity in Cygb-deficient mice compared with wild-type mice (arrows). Representative H\&E-stained paraffin sections (bottom) of hemangioma from wild-type and poorly differentiated hepatocellular carcinoma composed of small immature neoplastic cells with mitotic figures (the line indicates the boundary of tumor and nontumor areas), from $\mathrm{Cygh}^{-1-}$ mice. Scale bar $=100 \mu \mathrm{m}$ C: Determination of liver/body weight ratios, maximum (Max) tumor sizes, and liver tumor numbers for $C y g b^{+/+}$(white bars), $C y g b^{+/-}$ (gray bars), and $C y g b^{-/-}$(black bars) mice ( $n=$ 7 to 12 ). Values are given as the mean \pm SD. ${ }^{*} P<$ $0.05,{ }^{* *} P<0.01$. D: Representative gross photographs (top) and microphotographs (bottom) of lungs from $C y g b^{+/+}, C y g b^{+/-}$, and $C y g b^{-/-}$ DEN-treated mice. Lung tumors were only found in $\mathrm{Cygh}^{+/-}$and $\mathrm{Cygh}^{-/-}$mice. Arrows indicate lung tumors that were classified as squamous cell carcinomas. Scale bar $=100 \mu \mathrm{m}$. 
ment. $^{22,23}$ In this study, we examined whether the loss of Cygb could influence the toxicity and carcinogenesis of DEN in the tumor-resistant C57BL/6J strain. We administered 25-ppm DEN to the drinking water of Cygb-deficient male mice at the age of 4 weeks. Unexpectedly, as early as 25 weeks after DEN treatment, $100 \%$ of $\mathrm{Cygh}^{-1-}$ and $\mathrm{Cygh}^{+/-}$mice developed tumors in the liver and lungs, whereas the tumor incidence in wild-type mice was $44.4 \%$ (Figure 2, A and B). The liver/body weight ratio was approximately $50 \%$ more than in wild-type animals (Figure $2 \mathrm{C}$ ) because of these tumor masses. Cyg $b^{-1-}$ mice displayed significantly larger lesions and produced tumors more frequently than wild-type mice (Figure 2C).

DEN has induced tumors in the liver and in the lungs of the B6C3F1 mouse strain, although with a lower frequency. ${ }^{24}$ Consistent with these studies, lung tumors were found in $\mathrm{Cygh}^{+/-}$and $\mathrm{Cygh}^{-1-}$ mice, with a frequency of $11.7 \%$ and $57.1 \%$, respectively, whereas $\mathrm{Cygb}^{+/+}$mice showed complete resistance (Figure 2, A and D).

Next, using a low dose of DEN, at which wild-type mice do not develop liver tumors, we examined whether Cygb deficiency functioned as a tumor promoter. We took advantage of earlier observations that, when DEN was administered to mice at a nontoxic dose, it failed to induce liver cancer, unless combined with a tumor promoter, such as phenobarbital. ${ }^{25,26}$ We maintained wild-type and Cygb-deficient male mice during DEN administration at the nontoxic dose of $0.05 \mathrm{ppm}$ for 36 weeks. As a result, $>40 \%$ of $\mathrm{Cygh}^{-1-}$ mice developed tumors in the liver or lungs, whereas, as expected, wild-type mice exhibited no tumor formation (Figure 3). Cygh ${ }^{+/-}$mice showed an intermediate sensitivity. These findings confirm the tumor-promoting effects of Cygb depletion.

To define the histological features of the liver tumors, liver samples were evaluated $\mathrm{HC}$. The liver tumors in C57BL/6 mice (wild type) administered DEN in drinking water were dominantly composed of cholangiomas, hemangiomas, hemangiosarcomas, and, to a lesser degree, hepatocellular carcinomas. ${ }^{27-29}$ In this study, Cygb-deficient and wild-type mice developed similar histological alterations in the liver, including hemangioma (Figure 2B), hepatocellular carcinoma (Figures $2 \mathrm{~B}$ and $3 \mathrm{~B}$ ), which stained positive for alpha-fetoprotein (AFP) (Figure $4 \mathrm{~A}$ ), and cholangioma, which stained positive for CK19 (Figure 4A). Quantitative real-time PCR (RT-qPCR) analysis showed increased mRNA expression of AFP in $\mathrm{Cygh}^{+-}$and $\mathrm{Cygb}^{-1-}$ mice compared with their wildtype counterparts (Figure 4B).

In the liver, Cygb was present in stellate cells. ${ }^{1,4}$ We used cellular retinol-binding protein- 1 and $\alpha$-smooth muscle actin antibodies to detect stellate cell expression. The presence of cellular retinol-binding protein-1- and $\alpha$-smooth muscle actin-expressing cells in the liver parenchyma from DEN-treated homozygote mice indicated that stellate cells were present along sinusoids, even in the absence of Cygb (Figure 4C). The expression of $\alpha$-smooth muscle actin and desmin, another marker of stellate cells, was markedly augmented in $\mathrm{Cygh}^{-1-}$ mice (Figure 4C). Next, we assessed whether liver fibrosis developed in these mice. Sirius red staining for collagen deposition in paraffin-embedded sections of liver samples
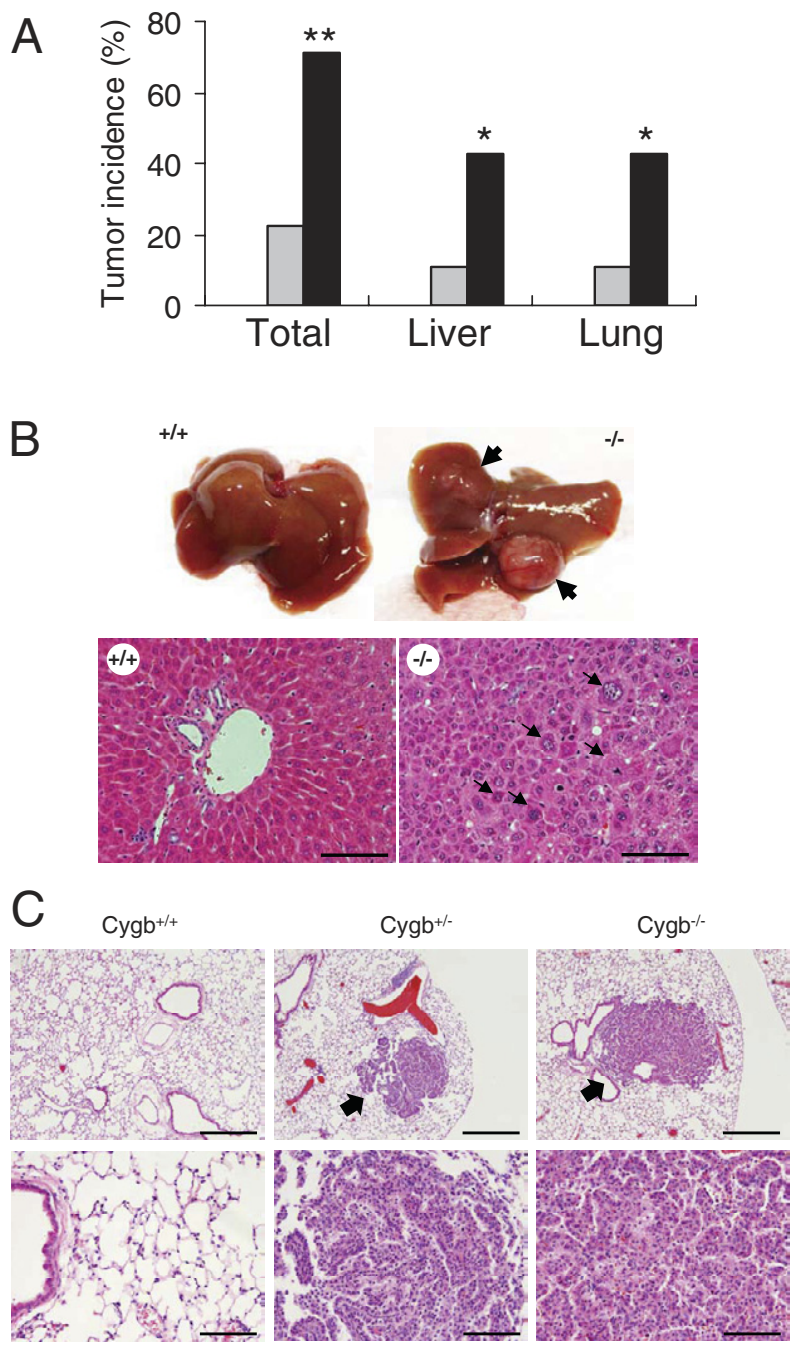

Figure 3. Tumor development in Cygb-deficient mice treated with 0.05-ppm DEN for 36 weeks. A: Tumor incidence in total, livers, and lungs from 0.05-ppm DEN-treated $\mathrm{Cygb}^{+/+}$(white bars), $\mathrm{Cygb}^{+/-}$(gray bars), and $\mathrm{Cyg}^{-1-}$ (black bars) mice $(n=7$ to 15$) .{ }^{*} P<0.05$, ${ }^{* * *} P<0.01$ compared with the wild type. B: Gross photographs of livers (top) from $C y g b^{+/+}$and $\mathrm{Cygh}^{-1-}$ mice treated with $0.05-\mathrm{ppm} \mathrm{DEN}$, as previously mentioned; arrows indicate lung tumors. Representative photomicrographs of H\&E-stained paraffin sections of liver parenchyma (bottom) from wild-type and moderately differentiated hepatocellular carcinoma composed of large cells that vary in size and shape (arrows), from $\mathrm{Cyg}^{-/-}$mice. Scale bar $=100 \mu \mathrm{m}$. C: Representative photomicrographs of H\&E-stained paraffin sections of lungs from $\mathrm{Cygb}^{+/+}, \mathrm{Cygb}^{+/-}$, and $C y g b^{-/-}$mice treated with $0.05-\mathrm{ppm}$ DEN for 36 weeks. Scale bars: $400 \mu \mathrm{m}$ (top); $100 \mu \mathrm{m}$ (bottom). Arrows indicate lung tumors that were classified as adenocarcinomas. No lung tumor was observed in $C y g b^{+/+}$mice.

collected from $\mathrm{Cygb}^{+/+}$and $\mathrm{Cygh}^{-1-}$ mice treated with 25-ppm DEN for 25 weeks showed marked deposition of collagen (red) around the hepatocytes (pericellular fibrosis) in Cygb $b^{-1-}$ mice (Figure 4D). Morphometric image analysis was performed with a computerized system, consisting of a photomicroscope, a digital camera, and LuminaVision 2.4 bioimaging software, to quantitatively assess fibrosis. The Sirius red-positive area of $\mathrm{Cygh}^{-1-}$ mice $(7.67 \% \pm 7.82 \%$, $n=3)$ was significantly greater than that in $\mathrm{Cygb}^{+/+}$mice (mean \pm SD, $1.36 \% \pm 0.36 \%, n=3 ; P<0.05$, KruskalWallis test). Pericellular fibrosis with collagen deposition was accompanied by significantly augmented mRNA expression of collagen $1 \alpha 1$ and tissue inhibitor of matrix met- 
A
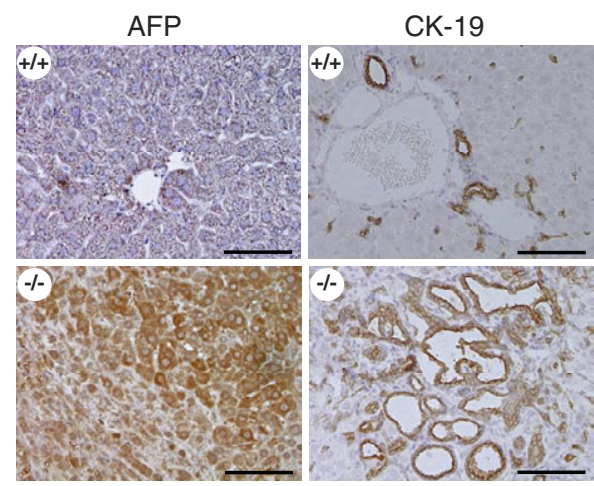

$\alpha$-SMA
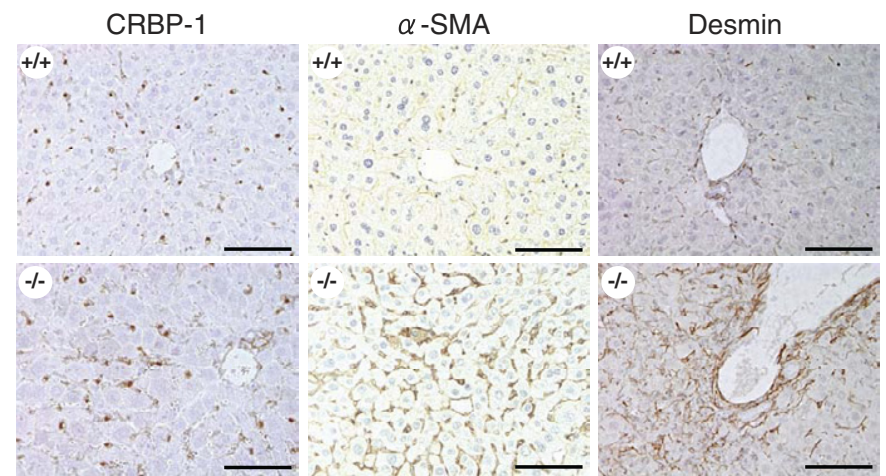

D

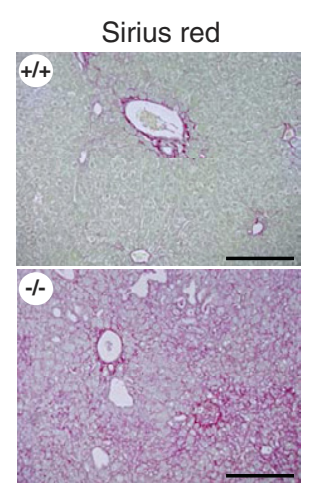

$E$

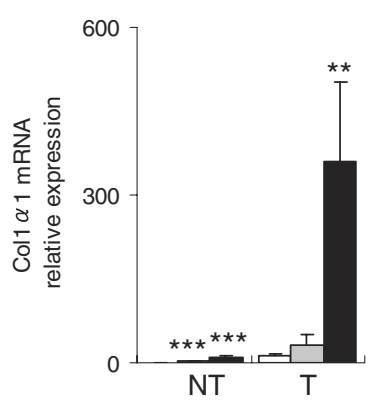

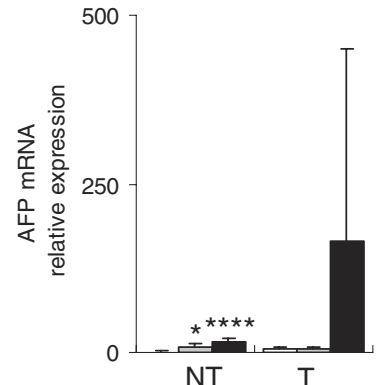

Figure 4. Expression of tumor markers and liver fibrosis development in DEN-treated Cygb deficient mice. Cygb-deficient mice from Figure 2 were subjected to histological and biochemical analyses. A: Paraffin-embedded liver sections from $\mathrm{Cygb}^{+/+}$and $\mathrm{Cygb}^{-/-}$mice were stained with AFP and cytokeratin 19 (CK-19). Scale bar $=100 \mu \mathrm{m}$. B: Expression of AFP mRNA in $\mathrm{Cygh}^{+/+}$(white bars), Cygb ${ }^{+/-}$(gray bars), and $\mathrm{Cygh}^{-1-}$ (black bars) mice livers was determined by RT-qPCR ( $n=7$ to 12). Levels were normalized to GAPDH. Values are given as the mean \pm SD of all experiments. NT, nontumor area; $\mathrm{T}$, liver tumor. ${ }^{*} P<0.05,{ }^{* * * *} P<0.0001$. C: Paraffin-embedded liver sections were IHC stained for the detection of CRBP-1, $\alpha$-SMA, and desmin. Scale bar $=100 \mu \mathrm{m}$. D and E: Development of liver fibrosis. D: Sirius red staining for collagen deposition in paraffin-embedded liver sections. Scale bar $=100 \mu \mathrm{m}$. There was marked deposition of collagen (red) around the hepatocytes (pericellular fibrosis) in $C y g b^{-/-}$mice. $\mathbf{E}$ Relative levels of collagen (Col) $1 \alpha 1$ and tissue inhibitor of matrix metalloproteinase-1 (TIMP-1) mRNA in the nontumor area (NT; $n=7$ to 12 ) and in liver tumors (T; $n=3$ to 5 ) of $C y g b^{+/+}$ (white bars), $\mathrm{Cygb}^{+/-}$(gray bars), and $\mathrm{Cygh}^{-/}$ (black bars) mice were determined by RT-qPCR and normalized to GAPDH mRNA. Values are given as the mean $\pm \mathrm{SD}$ of all experiments. ${ }^{*} P<$ $0.05,{ }^{* *} P<0.01$, and ${ }^{* * *} P<0.001$. taloproteinase- 1 in the livers of $\mathrm{Cyg}^{-1-}$ mice, compared with that of $\mathrm{Cygh}^{+/+}$mice treated with 25-ppm DEN for 25 weeks (Figure 4E). Thus, the augmented occurrence of pericellular fibrosis and fibrotic reactions in Cygb deficiency may be involved in the development of liver cancer.

\section{Cygb Loss Is Associated with Increased Cancer Cell Proliferation}

Two important cellular processes in tumorigenesis are cell apoptosis and proliferation. To examine how Cygb loss affected these two processes in the livers of C57BL/6J mice, we determined the labeling indexes of TUNEL, as a marker of apoptotic cells, and proliferating cell nuclear antigen (PCNA), as a marker of proliferating cells in the liver, both in the tumor and adjacent nontumor areas of $\mathrm{Cygh}^{+/+}$and $\mathrm{Cygh}^{-1-}$ mice treated with 25-ppm DEN in drinking water for 25 weeks. Liver tumors in Cygb ${ }^{-1-}$ mice exhibited reduced apoptotic cell death relative to liver tumors in $\mathrm{Cygh}^{+/+}$mice (Figure 5, A and C) and showed elevated mRNA expression of the antiapoptotic protein Bcl-2 (Figure $5 \mathrm{E})$. Liver tumors in the Cygb ${ }^{-1-}$ mice exhibited more proliferating cells than the tumors of the wild-type mice, as shown by PCNA labeling (Figure 5, B and D), in addition to elevated cyclin D1 mRNA expression (Figure 5F). These results suggest that stimulation of proliferating neoplastic cells is the primary cellular mechanism for increased liver tumorigenesis in Cygb-deficient mice.

\section{Cygb-Deficient Mice Exhibit Elevated Phosphorylated Akt and Erk and Liver Inflammation}

To identify the signaling pathways responsible for enhanced hepatocyte survival and proliferation, we ex- 
A
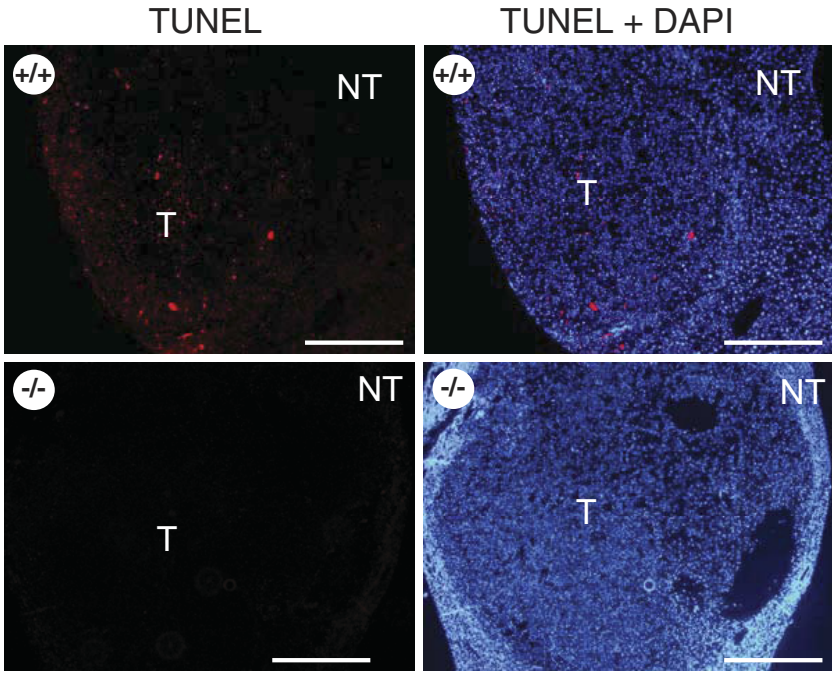

B

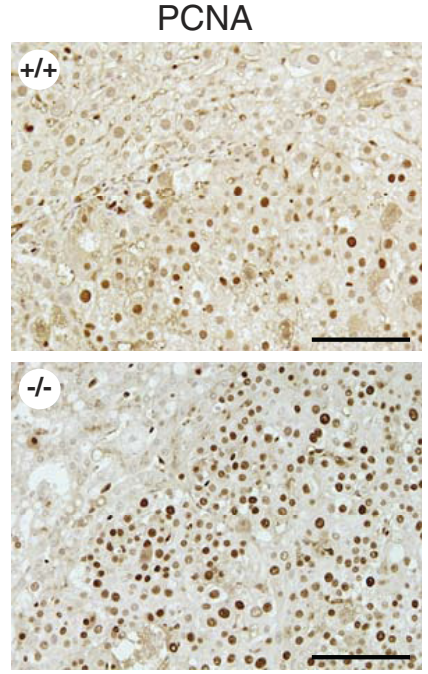

C

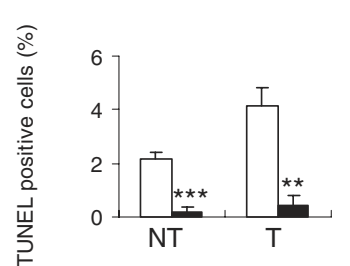

D

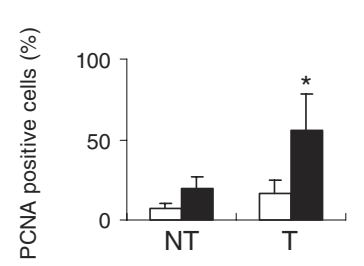

E

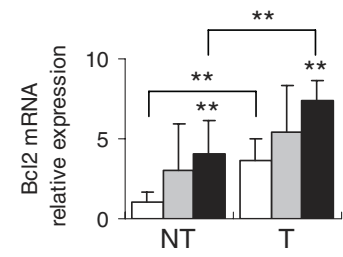

$\mathrm{F}$

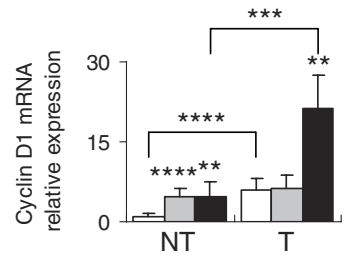

Figure 5. Tumor-bearing Cygb-deficient mice are associated with increased cancer cell proliferation and reduced apoptosis. A: Paraffin-embedded liver sections from wild-type $(+/+)$ and homozygous $(-/-)$ mice treated with 25 -ppm DEN for 25 weeks were TUNEL labeled (left) and counterstained with DAPI (right). Apoptotic cells were present in the tumor (T) area in wild-type mice. NT, nontumor area. Scale bar $=400 \mu \mathrm{m}$. B: Paraffin-embedded liver sections were stained with PCNA. Scale bar $=100 \mu \mathrm{m}$. The frequency of apoptotic $(\mathbf{C})$ or proliferate (D) cells was determined by counting TUNEL- or PCNA-positive cells, respectively (cells with the nucleus stained dark brown in the case of PCNA), in at least 1000 cells from each liver $(n=3$ ). Expression levels of Bcl-2 (E) and cyclin D1 (F) mRNA in the NT ( $n=7$ to 12$)$ and T ( $n=3$ to 5 ) areas from 25 -ppm DEN-treated mice were determined by RT-qPCR and normalized to GAPDH. Cyg $b^{+/+}$(white bars), Cyg $b^{+/-}$(gray bars), and Cyg $b^{-1-}$ (black bars) mice. Values are given as the mean \pm SD of all experiments. ${ }^{*} P<0.05,{ }^{* *} P<0.01,{ }^{* * * *} P<0.001$, and ${ }^{* * * * *} P<0.0001$.

amined the effects of Cygb deficiency on the major pathways implicated in liver cancer. ${ }^{30}$ As expected, Cygb loss was associated with an increase in both Akt phosphorylation and abundance in the livers (Figure $6 A)$. This observation was also evident for Erk signaling (Figure 6, A and B). Consistent with increased Erk phosphorylation, Cygb-deficient mice exhibited increased expression of cyclin D1 (Figure 6A), and Jun and Fos mRNA in nontumor and tumor areas, relative to wild type (Figure 6C). These results suggested that the Akt and Erk pathways are activated in response to Cygb deficiency. The increased levels of Akt and Erk activation correlated with a marked elevation of IL-6 mRNA in both nontumor and tumor areas in Cygbdeficient mice (Figure 6D). IL-6 is a tumor-promoting cytokine that is required for Erk activation and contributes to alterations in Akt signaling. ${ }^{31}$ Knowing that IL-6 functions as a downstream mediator for both IL-1 and tumor necrosis factor $\alpha,{ }^{32}$ we examined the expression of these two cytokines. Remarkably, IL-1 $\beta$ and $\operatorname{Tnf} \alpha$ levels (Figure 6D) increased 10- and 30-fold, respectively, at the mRNA level in the nontumor area of the liver and increased further in the tumors, relative to wild type. Cygb-deficient mice also had increased expression of Tgf $\beta 3$ mRNA (Figure 6D). These data suggest that Cygb loss can trigger inflammation and lead to the long-term elevation of tumor-promoting cytokines, resulting in the development of tumors.

\section{Nitrotyrosine Accumulation in the Livers of DEN-Treated Cygb-Deficient Mice}

Long-term administration of DEN has induced the expression of the inducible isoform of NO synthase and 3-nitrotyrosine, a marker of peroxynitrite formation, in preneoplastic and neoplastic rat liver tissues. ${ }^{33}$ In this study, we detected the overproduction of nitrotyrosine in tumor and nontumor liver tissues of Cygb-deficient mice, compared with wild-type mice, as shown by IHC (Figure 7A) and immunoblot analyses (Figure 7B). These results indicate the high production of $\mathrm{NO}$, together with superoxide, in Cygb-deficient mice.

\section{Dysregulation of Genes Associated with Cell Proliferation and Differentiation in Cygb-Deficient Mice}

To further screen for cellular alterations caused by Cygb gene disruption, we compared gene expression profiles between Cygb-deficient mice and their wild-type counterparts after 25-ppm DEN treatment for 25 weeks. We 
observed the altered expression of cancer genes, including p53, cyclin D2, Pak1 (p21-activated kinase), Src, Cdkn2a, and Cebpa (CCAAT/enhancer-binding protein $\alpha$ ) (data not shown). We examined in detail the mRNA levels of these genes by RT-qPCR. Consistent with the high rate of cellular proliferation in the liver of Cygb-deficient mice (Figure 5, B and D), we observed overexpression of cyclin D2 (Figure 8A) and p53 (Figure 8B), which have displayed high expression in astrocytomas, a type of brain tumor. ${ }^{34}$ Pak1 promotes malignant tumor progression, and the Src proto-oncogene has shown increased expression in human skin tumors and leukemia. ${ }^{35-38}$ In this study, we found that the mRNA expression of Pak1, in addition to Src, increased fivefold in the livers of $\mathrm{Cygh}^{-1-}$ mice, relative to the wild type (Figure 8, C and D).

Cdkn2a, a tumor suppressor that negatively regulates the cell cycle, displayed increased expression at the mRNA level in the livers of Cygb-deficient mice (Figure 8E), consistent with other studies ${ }^{39,40}$ on sarcomas and lung tumors.

Cebpa has the ability to inhibit proliferation, particularly in hepatocytes. ${ }^{41,42}$ Down-regulation of CEBPA has been

A
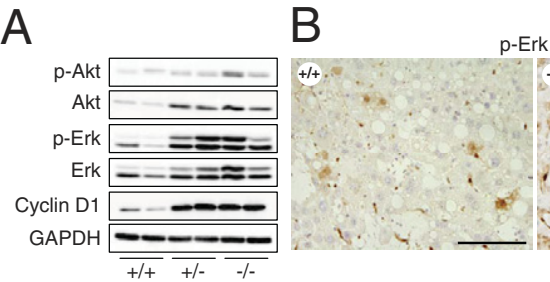

C
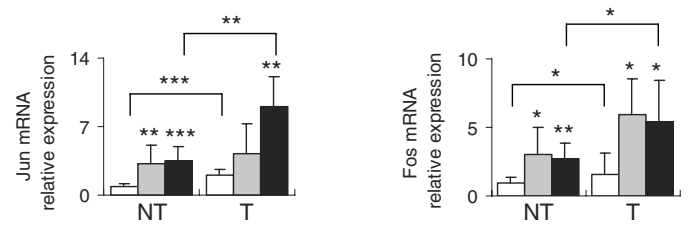

$\mathrm{D}$
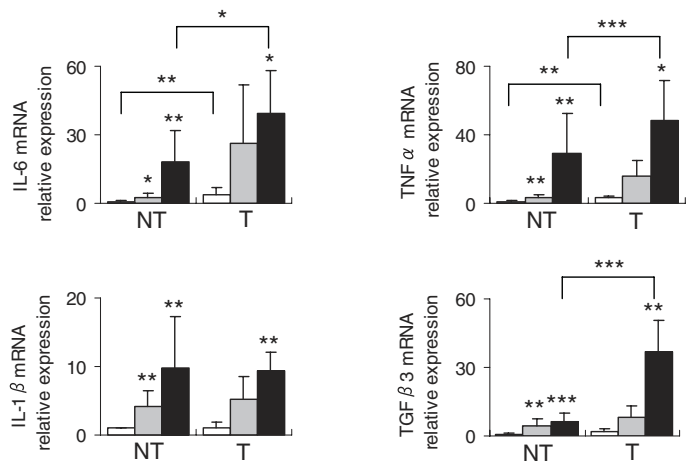

Figure 6. Tumor-bearing Cygb-deficient mice exhibited elevated phosphorylated Akt (p-Akt), phosphorylated Erk (p-Erk), and inflammation. Cygb deficient mice from Figure 2 were subjected to additional biochemical and gene expression analyses. A: The liver was lysed and gel separated, and the levels of p-Akt, p-Erk, total Akt, total Erk, and cyclin D1 were examined by immunoblot analyses. All blots were reprobed with anti-GAPDH as a loading control. B: Paraffin-embedded liver sections were stained for phosphorylated Erk. Scale bar $=100 \mu \mathrm{m}$. $\mathbf{C}$ and D: Relative mRNA levels of Jun and Fos $(\mathbf{C})$ and IL-6, $\operatorname{Tnf} \alpha$, IL- $1 \beta$, and $\operatorname{Tgf} \beta 3$ (D) in the nontumor (NT; $n=7$ to 12 ) and tumor (T; $n=3$ to 5 ) areas of the liver were determined by RT-qPCR and normalized to GAPDH. $\mathrm{Cygb}^{+/+}$(white bars), $\mathrm{Cygb}^{+/-}$(gray bars), and $\mathrm{Cygh}^{-1-}$ (black bars) mice. Values are given as the mean $\pm \mathrm{SD}$ of all experiments. ${ }^{*} P<0.05,{ }^{* *} P<0.01$, and ${ }^{* * * * *} P<0.001$.
A

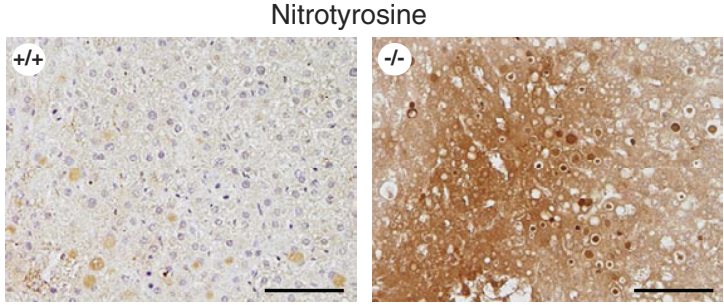

B

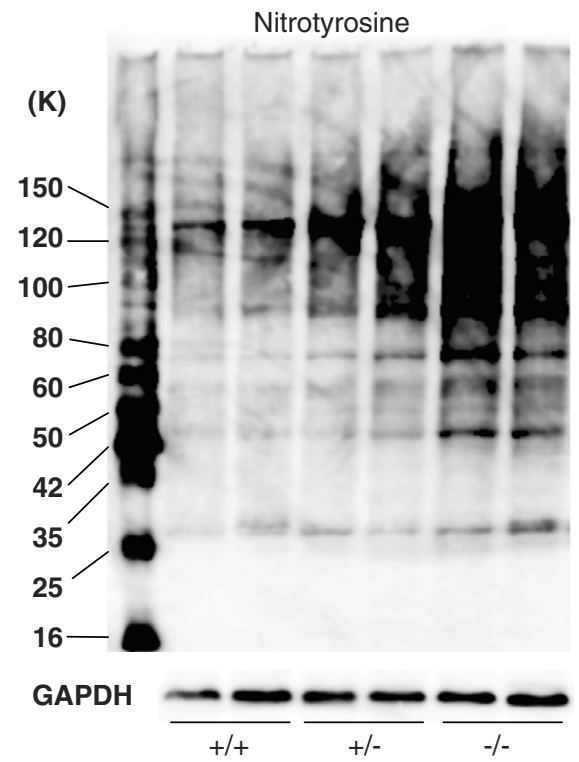

Figure 7. Peroxynitrite formation in the livers of $C y g b$-deficient mice. A: Paraffinembedded liver sections from wild-type $(+/+)$ and homozygous $(-/-)$ mice treated with 25 -ppm DEN for 25 weeks were stained with antinitrotyrosine. Nitrotyrosine-containing proteins were strongly expressed in the cytoplasm and nuclei of cancer cells in the tumor area, particularly in the inclusions of cancer cells. Scale $\mathrm{bar}=100 \mu \mathrm{m}$. B: Protein homogenates of liver tissues from $\mathrm{Cygh}^{+++}, \mathrm{Cygb}^{+/-}$, and $\mathrm{Cygh}^{-/-}$mice treated with 25-ppm DEN for 25 weeks were subjected to immunoblot detection for nitrotyrosine. GAPDH was used as a loading control. K, kDa (molecular mass)

reported in human myeloid leukemia. ${ }^{43}$ Consistent with these studies, our results showed decreased expression of Cebpa mRNA in the liver of Cygb-deficient mice, relative to the wild type (Figure 8F).
A

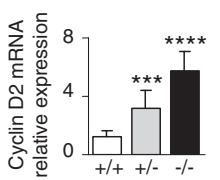

$\mathrm{D}$

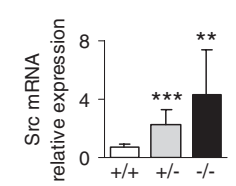

B

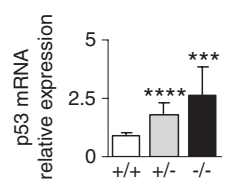

$E$

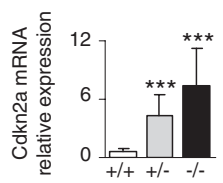

C

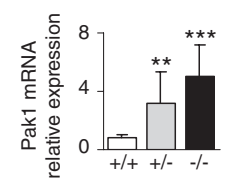

$\mathrm{F}$

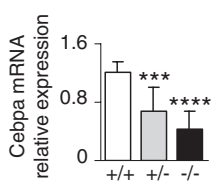

Figure 8. Altered regulation of cancer genes in Cygb-deficient mice. RT-qPCR analysis of cell growth-related gene transcripts cyclin D2 (A), p53 (B), and Pak1 (C) and cell differentiation and apoptosis-related gene transcripts Src (D), Cdkn2a $(\mathbf{E})$, and Cebpa $(\mathbf{F})$ in liver from mice treated with 25-ppm DEN for 25 weeks ( $n=7$ to 12). Levels are normalized to GAPDH. Cygb ${ }^{++}$(white bars) $\mathrm{Cygb}^{+/-}$(gray bars), and $\mathrm{Cygh}^{-1-}$ (black bars) mice. Values are given as the mean $\pm \mathrm{SD}$ of all experiments. ${ }^{* * *} P<0.01,{ }^{* * * *} P<0.001$, and ${ }^{* * * * * *} P<0.0001$. 


\section{Discussion}

In the present study, we showed that loss of Cygb in C57BL/6J mice markedly increased their susceptibility to DEN-induced tumorigenesis. In the absence of Cygb, liver tumors developed earlier, were larger, and were more numerous compared with wild-type mice. By administering low-dose DEN to adult mice (0.05 ppm), which failed to induce liver cancer in wild-type mice, we observed the tumor-promoting effects of Cygb deficiency.

We observed high levels of Cygb expression in hepatic stellate cells, a liver-specific pericyte, from which Cygb was originally discovered by proteomic analysis of primary cultured rat cells and other stromal cells in the visceral organs, including the pancreas, gut, spleen, lung, and kidney. ${ }^{1,4}$ These stromal cells are pericytes localized around the capillaries of the organs that are capable of vitamin A storage. Thus, we propose that Cygb may be an indicator of a vitamin A-storing phenotype of myofibroblasts of endoderm origin. However, as previously reported, , ${ }^{1,4,5}$ Cygb is ubiquitously expressed in all body organs in human, as in mice and rats. At the mRNA level, high expression is evident in the adult human heart and liver; modest expression is evident in the brain, kidney, trachea, and placenta; and low expression is evident in the adult skeletal muscle. ${ }^{44}$ Several cancer cell lines, including HepG2 cells, ${ }^{44}$ the $\mathrm{NCl}-\mathrm{H} 2228$ lung cancer cell line, and HCC 1569 breast cancer cells, ${ }^{19}$ also display CYGB mRNA expression. These observations indicate the role of Cygb in the regulation of cellular function originating from the epithelia. In this context, cancer development in the liver and lungs in Cygb ${ }^{-1-}$ mice is anticipated. However, the role of mesenchymal cells that express Cygb highly in tumor development should be further evaluated because these myofibroblasts represent important environmental factors during tumor formation.

Cygb expression is augmented under hypoxia in the liver, heart, brain, and skeletal muscle and in HN33 cells (an immortalized mouse hippocampal cell line), BEAS-2B cells (a transformed human bronchial epithelial cell line), and HeLa cells (a human cervix carcinoma cell line). ${ }^{16}$ Overexpression of Cygb protects mouse neuroblastoma $\mathrm{N} 2 \mathrm{a}$ cells and human neuroblastoma SH-SY5Y cells under $\mathrm{H}_{2} \mathrm{O}_{2}$ exposure and the human neuronal cell line TE671 under prooxidant Ro19-8022 stimulation. ${ }^{11,12,44}$ Overexpression of Cygb in the liver, induced by adenoassociated-virus-induced transfection, protects the liver from oxidative injury. ${ }^{13}$ Conversely, the role of Cygb as an $\mathrm{NO}$ scavenger in rat hepatocytes and NIH3T3 fibroblasts may protect cells from $\mathrm{NO}$-induced toxicity. ${ }^{8,33}$ These reports and our findings regarding the accumulation of nitrotyrosine protein adducts in Cygb-deficient mice indicate the cytoprotective and antioxidative properties of Cygb.

Several tumor suppressor genes are located in both arms of chromosome 17. TP53, a known tumor suppressor gene at $17 \mathrm{p} 13.1$, is one of the most frequently mutated genes in cancers, including hepatocellular carcinoma. ${ }^{45}$ BRCA1 (breast cancer 1, early onset) at $17 \mathrm{q} 12$ is a human tumor suppressor gene encoding the breast cancer type 1 susceptibility protein, ${ }^{46}$ which is present in the breast and other tissues, aiding the repair of dam- aged DNA and the destruction of the cell when DNA cannot be repaired. If BRCA1 is damaged, cells duplicate uncontrollably, leading to cancer. Other known breast cancer-associated genes include septin, DMC1, and HER2/ErbB2. ${ }^{47,48}$ Because CYGB exists on chromosome 17q25, genes on this chromosome appear commonly in the tumorigenesis of epithelial cells and mutation or epigenetic modification of these genes appears to trigger malignant transformation.

Clinically, liver cancer develops from a fibrotic liver, with chronic trauma induced by alcohol abuse and hepatitis virus $\mathrm{B} / \mathrm{C}$ infection. ${ }^{49}$ Chronic inflammation offers an appropriate environment for cancer development, by producing multiple growth factors, extracellular matrices, and neovascularization involving local hypoxia. ${ }^{50}$ In this context, the augmented occurrence of pericellular fibrosis and fibrotic reactions (Figure 4, C-E) in Cygb deficiency may be involved in the development of liver cancer.

In conclusion, to our knowledge, this is the first report that Cygb deficiency induces susceptibility to cancer development in the liver and lungs of mice receiving DEN treatment. Thus, Cygb-deficient mice may provide a useful animal model to study cancer development in the liver and lungs; globins, such as Cygb, may shed new light on the biological features of organ carcinogenesis.

\section{Acknowledgment}

We thank Drs. Masaru Enomoto, Hideki Fujii, and Thoru Komiya for their valuable comments during this study.

\section{References}

1. Kawada N, Kristensen DB, Asahina K, Nakatani K, Minamiyama Y Seki S, Yoshizato K: Characterization of a stellate cell activationassociated protein (STAP) with peroxidase activity found in rat hepatic stellate cells. J Biol Chem 2001, 276:25318-25323

2. Burmester T, Ebner B, Weich B, Hankeln T: Cytoglobin: a novel globin type ubiquitously expressed in vertebrate tissues. Mol Biol Evol 2002, 19:416-421

3. Sawai H, Kawada N, Yoshizato K, Nakajima H, Aono S, Shiro Y: Characterization of the heme environmental structure of cytoglobin, a fourth globin in humans. Biochemistry 2003, 42:5133-5142

4. Nakatani K, Okuyama H, Shimahara Y, Saeki S, Kim DH, Nakajima Y, Seki S, Kawada N, Yoshizato K: Cytoglobin/STAP, its unique localization in splanchnic fibroblast-like cells and function in organ fibrogenesis. Lab Invest 2004, 84:91-101

5. Shigematsu A, Adachi Y, Matsubara J, Mukaide H, Koike-Kiriyama N, Minamino K, Shi M, Yanai S, Imamura M, Taketani S, Ikehara S: Analyses of expression of cytoglobin by immunohistochemical studies in human tissues. Hemoglobin 2008, 32:287-296

6. Sugimoto $H$, Makino M, Sawai $H$, Kawada N, Yoshizato $K$, Shiro $Y$ : Structural basis of human cytoglobin for ligand binding. J Mol Biol 2004, 339:873-885

7. Li RC, Lee SK, Pouranfar F, Brittian KR, Clair HB, Row BW, Wang Y, Gozal D: Hypoxia differentially regulates the expression of neuroglobin and cytoglobin in rat brain. Brain Res 2006, 1096:173-179

8. Fordel E, Geuens E, Dewilde S, Rottiers P, Carmeliet P, Grooten J, Moens L: Cytoglobin expression is upregulated in all tissues upon hypoxia: an in vitro and in vivo study by quantitative real-time PCR. Biochem Biophys Res Commun 2004, 319:342-348

9. Guo X, Philipsen S, Tan-Un KC: Study of the hypoxia-dependent regulation of human CYGB gene. Biochem Biophys Res Commun 2007, 364:145-150 
10. Hodges NJ, Innocent N, Dhanda S, Graham M: Cellular protection from oxidative DNA damage by over-expression of the novel globin cytoglobin in vitro. Mutagenesis 2008, 23:293-298

11. Fordel E, Thijs L, Martinet W, Lenjou M, Laufs T, Van Bockstaele D, Moens L, Dewilde S: Neuroglobin and cytoglobin overexpression protects human SH-SY5Y neuroblastoma cells against oxidative stress-induced cell death. Neurosci Lett 2006, 410:146-151

12. Fordel E, Thijs L, Martinet W, Schrijvers D, Moens L, Dewilde S: Anoxia or oxygen and glucose deprivation in SH-SY5Y cells: a step closer to the unraveling of neuroglobin and cytoglobin functions. Gene 2007, 398:114-122

13. Xu R, Harrison PM, Chen M, Li L, Tsui TY, Fung PC, Cheung PT, Wang G, Li H, Diao Y, Krissansen GW, Xu S, Farzaneh F: Cytoglobin overexpression protects against damage-induced fibrosis. Mol Ther 2006, 13:1093-1100

14. McRonald FE, Liloglou T, Xinarianos G, Hill L, Rowbottom L, Langan JE, Ellis A, Shaw JM, Field JK, Risk JM: Down-regulation of the cytoglobin gene, located on 17q25, in tylosis with oesophageal cancer (TOC): evidence for trans-allele repression. Hum Mol Genet 2006, 15:1271-1277

15. Xinarianos G, McRonald FE, Risk JM, Bowers NL, Nikolaidis G, Field JK, Liloglou T: Frequent genetic and epigenetic abnormalities contribute to the deregulation of cytoglobin in non-small cell lung cancer. Hum Mol Genet 2006, 15:2038-2044

16. Shaw RJ, Omar MM, Rokadiya S, Kogera FA, Lowe D, Hall GL, Woolgar JA, Homer J, Liloglou T, Field JK, Risk JM: Cytoglobin is upregulated by tumour hypoxia and silenced by promoter hypermethylation in head and neck cancer. Br J Cancer 2009, 101: $139-144$

17. Presneau N, Dewar K, Forgetta V, Provencher D, Mes-Masson AM, Tonin PN: Loss of heterozygosity and transcriptome analyses of a 1.2 $\mathrm{Mb}$ candidate ovarian cancer tumor suppressor locus region at 17q25.1-q25.2. Mol Carcinog 2005, 43:141-154

18. Chua PJ, Yip GW, Bay BH: Cell cycle arrest induced by hydrogen peroxide is associated with modulation of oxidative stress related genes in breast cancer cells. Exp Biol Med (Maywood) 2009, 234: 1086-1094

19. Shivapurkar N, Stastny V, Okumura N, Girard L, Xie Y, Prinsen C, Thunnissen FB, Wistuba II, Czerniak B, Frenkel E, Roth JA, Liloglou T, Xinarianos G, Field JK, Minna JD, Gazdar AF: Cytoglobin, the newest member of the globin family, functions as a tumor suppressor gene. Cancer Res 2008, 68:7448-7456

20. Sinal CJ, Tohkin M, Miyata M, Ward JM, Lambert G, Gonzalez FJ: Targeted disruption of the nuclear receptor FXR/BAR impairs bile acid and lipid homeostasis. Cell 2000, 102:731-744

21. Verna L, Whysner J, Williams GM: N-nitrosodiethylamine mechanistic data and risk assessment: bioactivation, DNA-adduct formation, mutagenicity, and tumor initiation. Pharmacol Ther 1996, 71:57-81

22. Diwan BA, Rice JM, Ohshima M, Ward JM: Interstrain differences in susceptibility to liver carcinogenesis initiated by $\mathrm{N}$-nitrosodiethylamine and its promotion by phenobarbital in C57BL/6NCr, C3H/ HeNCrMTV- and DBA/2NCr mice. Carcinogenesis 1986, 7:215-220

23. Drinkwater NR, Ginsler JJ: Genetic control of hepatocarcinogenesis in C57BL/6J and $\mathrm{C} 3 \mathrm{H} / \mathrm{HeJ}$ inbred mice. Carcinogenesis 1986, 7:1701-1707

24. Vesselinovitch SD, Koka M, Mihailovich N, Rao KV: Carcinogenicity of diethylnitrosamine in newborn, infant, and adult mice. J Cancer Res Clin Oncol 1984, 108:60-65

25. Gray R, Peto R, Brantom P, Grasso P: Chronic nitrosamine ingestion in 1040 rodents: the effect of the choice of nitrosamine, the species studied, and the age of starting exposure. Cancer Res 1991, 51: 6470-6491

26. Ward JM, Diwan BA, Ohshima M, Hu H, Schuller HM, Rice JM: Tumor-initiating and promoting activities of di(2-ethylhexyl) phthalate in vivo and in vitro. Environ Health Perspect 1986, 65:279-291

27. Iwai S, Murai T, Makino S, Min W, Morimura K, Mori S, Hagihara A, Seki S, Fukushima S: High sensitivity of fatty liver Shionogi (FLS) mice to diethylnitrosamine hepatocarcinogenesis: comparison to $\mathrm{C} 3 \mathrm{H}$ and C57 mice. Cancer Lett 2007, 246:115-121

28. Koen H, Pugh TD, Goldfarb S: Hepatocarcinogenesis in the mouse: combined morphologic-stereologic studies. Am J Pathol 1983, 112 $89-100$
29. Koen H, Pugh TD, Nychka D, Goldfarb S: Presence of alpha-fetoprotein-positive cells in hepatocellular foci and microcarcinomas induced by single injections of diethylnitrosamine in infant mice. Cancer Res 1983, 43:702-708

30. Whittaker S, Marais R, Zhu AX: The role of signaling pathways in the development and treatment of hepatocellular carcinoma. Oncogene 2010, 29:4989-5005

31. Grivennikov SI, Greten FR, Karin M: Immunity, inflammation, and cancer. Cell 2010, 140:883-899

32. Kamimura D, Ishihara K, Hirano T: IL-6 signal transduction and its physiological roles: the signal orchestration model. Rev Physiol Biochem Pharmacol 2003, 149:1-38

33. Ahn B, Han BS, Kim DJ, Ohshima H: Immunohistochemical localization of inducible nitric oxide synthase and 3-nitrotyrosine in rat liver tumors induced by $\mathrm{N}$-nitrosodiethylamine. Carcinogenesis 1999, 20: 1337-1344

34. Kheirollahi M, Mehr-Azin M, Kamalian N, Mehdipour P: Expression of cyclin D2, P53, Rb and ATM cell cycle genes in brain tumors. Med Oncol 2011, 28:7-14

35. Kumar R, Gururaj AE, Barnes CJ: p21-activated kinases in cancer Nat Rev Cancer 2006, 6:459-471

36. Siu MK, Wong ES, Chan HY, Kong DS, Woo NW, Tam KF, Ngan HY, Chan QK, Chan DC, Chan KY, Cheung AN: Differential expression and phosphorylation of Pak1 and Pak2 in ovarian cancer: effects on prognosis and cell invasion. Int J Cancer 2010, 127:21-31

37. Barnekow A, Paul E, Schartl M: Expression of the c-src proto-oncogene in human skin tumors. Cancer Res 1987, 47:235-240

38. McClain KL: Expression of oncogenes in human leukemias. Cancer Res 1984, 44:5382-5389

39. Maelandsmo GM, Berner JM, Florenes VA, Forus A, Hovig E, Fodstad O, Myklebost O: Homozygous deletion frequency and expression levels of the CDKN2 gene in human sarcomas: relationship to amplification and mRNA levels of CDK4 and CCND1. Br J Cancer 1995, 72:393-398

40. Belinsky SA, Swafford DS, Middleton SK, Kennedy CH, Tesfaigzi J: Deletion and differential expression of p16INK4a in mouse lung tumors. Carcinogenesis 1997, 18:115-120

41. Timchenko NA, Wilde M, Nakanishi M, Smith JR, Darlington GJ: CCAAT/enhancer-binding protein alpha (C/EBP alpha) inhibits cell proliferation through the p21 (WAF-1/CIP-1/SDI-1) protein. Genes Dev 1996, 10:804-815

42. Diehl AM, Johns DC, Yang S, Lin H, Yin M, Matelis LA, Lawrence JH: Adenovirus-mediated transfer of CCAAT/enhancer-binding proteinalpha identifies a dominant anti-proliferative role for this isoform in hepatocytes. J Biol Chem 1996, 271:7343-7350

43. Pabst T, Mueller BU, Harakawa N, Schoch C, Haferlach T, Behre G Hiddemann W, Zhang DE, Tenen DG: AML1-ETO downregulates the granulocytic differentiation factor C/EBPalpha in $\mathrm{t}(8 ; 21)$ myeloid leukemia. Nat Med 2001, 7:444-451

44. Asahina K, Kawada N, Kristensen DB, Nakatani K, Seki S, Shiokawa M, Tateno C, Obara M, Yoshizato K: Characterization of human stellate cell activation-associated protein and its expression in human liver. Biochem Biophys Acta 2002, 1577:471-475

45. Caron de Fromentel C, Soussi T: TP53 tumor suppressor gene: a model for investigating human mutagenesis. Genes Chromosomes Cancer 1992, 4:1-15

46. Deng CX: BRCA1: cell cycle checkpoint, genetic instability, DNA damage response and cancer evolution. Nucleic Acids Res 2006, 34:1416-1426

47. Harada $H$, Nagai $H$, Tsuneizumi M, Mikami I, Sugano S, Emi M Identification of DMC1, a novel gene in the TOC region on 17q25.1 that shows loss of expression in multiple human cancers. J Hum Genet 2001, 46:90-95

48. Coussens L, Yang-Feng TL, Liao YC, Chen E, Gray A, McGrath J, Seeburg PH, Libermann TA, Schlessinger J, Francke U, Levinson A, Ullrich A: Tyrosine kinase receptor with extensive homology to EGF receptor shares chromosomal location with neu oncogene. Science 1985, 230:1132-1139

49. Llovet JM, Burroughs A, Bruix J: Hepatocellular carcinoma. Lancet 2003, 362:1907-1917

50. Brunt EM: Pathology of nonalcoholic fatty liver disease. Nat Rev Gastroenterol Hepatol 2010, 7:195-203 